\title{
SEMIREGULAR INVARIANT MEASURES ON ABELIAN GROUPS
}

\author{
ANDRZEJ PELC
}

\begin{abstract}
A nonnegative countably additive, extended real-valued measure is called semiregular if every set of positive measure contains a set of positive finite measure. V. Kannan and S. R. Raju [3] stated the problem of whether every invariant semiregular measure defined on all subsets of a group is necessarily a multiple of the counting measure. We prove that the negative answer is equivalent to the existence of a real-valued measurable cardinal.

It is shown, moreover, that a counterexample can be found on every abelian group of real-valued measurable cardinality.
\end{abstract}

We consider countably additive, nonnegative extended real-valued measures which are not identically equal to zero. Such a measure is called universal on a set $X$ if it is defined on all subsets of $X$ and it is called semiregular if every set of positive measure contains a set of positive finite measure. A universal measure $m$ on a group $(G, \circ)$ is invariant if $m(a \circ A)=m(A)$ for every $a \in G, A \subset G$.

A measure is called $\kappa$-additive if the union of less than $\kappa$ sets of measure 0 has measure 0 . A cardinal $\kappa$ is called real-valued measurable if there exists a finite universal $\kappa$-additive measure on $\kappa$ which vanishes on singletons. ${ }^{1}$ It is well known that the existence of a real-valued measurable cardinal is unprovable in usual set theory with choice.

Erdös and Mauldin [2] proved that if $(G, \circ)$ is an uncountable group then there is no $\sigma$-finite invariant universal measure on $(G, \circ)$. Kannan and Raju [3] asked whether every invariant semiregular universal measure on a group is necessarily a multiple of the counting measure. It is clear that any measure providing a counterexample has to vanish on singletons.

We give the following solution to the above problem.

THEOREM 1. The following are equivalent:

(*) Every universal invariant semiregular measure on a group is a multiple of the counting measure.

(**) There does not exist a real valued measurable cardinal.

Proof. ${ }^{2}$ Assume that $(*)$ does not hold. Hence there exists a universal invariant semiregular measure on a group, vanishing on singletons. Let $A$ be any set of

Received by the editors July 1, 1981.

1980 Mathematics Subject Classification. Primary 43A05; Secondary 28C10, 03 E55.

Key words and phrases. Universal invariant measure, group, real-valued measurable cardinal.

'According to the common habit in modern set theory we identify every ordinal with the set of its predecessors and cardinals with initial ordinals.

${ }^{2}$ The present version of the proof is simpler than the original one thanks to the referee's suggestion. 
positive finite measure. It is well known (cf. [1]) that the minimal cardinality of a subset $B \subset A$ of positive measure is a real-valued measurable cardinal. Assume that $(* *)$ is false and let $\kappa$ be a real-valued measurable cardinal with a $\kappa$-additive universal measure $\mu$ on $\kappa$ vanishing on singletons and such that $\mu(\kappa)=1$. Let $(G, \circ)$ be the direct sum of $\kappa$ copies of the two-element group $C_{2}$. It will be convenient to view $(G, \circ)$ as the group of all finite subsets of $\kappa$ with symmetric difference as the group operation.

For each $p \in G$ set $G_{p}=\{q \in G: \exists \beta>\max (p)[q=p \cup\{\beta\}]\}$. Notice that the sets $G_{p}$ together with $\{\varnothing\}$ form a partition of $G$. Define $m: \mathcal{P}(G) \rightarrow R \cup\{+\infty\}$ by

$$
m(A)=\sum_{p \in G} \mu\left(\cup\left[G_{p} \cap A\right]\right),
$$

the right-hand sum being the upper bound of appropriate sums over finite subsets of G.

Clearly $m(\{p\})=0$ for each $p \in G$. If $m(A)>0$ then there is some $p \in G$ such that $\mu\left(\cup\left[G_{p} \cap A\right]\right)>0$. Of course, $A \cap G_{p} \subset A$ and $0<m\left(A \cap G_{p}\right)=$ $\mu\left(\cup\left[G_{p} \cap A\right]\right)<\infty$. Hence $m$ is semiregular.

We check that $m$ is $\kappa$-additive. Suppose $\left\{A_{\gamma}: \gamma<\beta\right\}$ is a pairwise disjoint family of subsets of $G$, with $\beta<\kappa$. Then for every $p \in G$ the sets $\cup\left(G_{p} \cap A_{\gamma}\right): \gamma<\beta$ are pairwise almost disjoint and we get

$$
\begin{aligned}
m\left(\bigcup_{\gamma<\beta} A_{\gamma}\right) & =\sum_{p \in G} \mu\left(\bigcup\left[G_{p} \cap\left(\bigcup_{\gamma<\beta} A_{\gamma}\right)\right]\right) \\
& =\sum_{p \in G} \mu\left(\bigcup_{\gamma<\beta} \cup\left[G_{p} \cap A_{\gamma}\right]\right) \\
& =\sum_{\gamma<\beta} \sum_{p \in G} \mu\left(\bigcup\left[G_{p} \cap A_{\gamma}\right]\right)=\sum_{\gamma<\beta} m\left(A_{\gamma}\right) .
\end{aligned}
$$

We now check that $m$ is invariant. Let $q \in G$ and $A \subset G$.

$$
\begin{aligned}
m(q \circ A) & =\sum_{p \in G} \mu\left(\bigcup\left[G_{p} \cap q \circ A\right]\right) \\
& =\sum_{p \in G} \mu\left(\bigcup\left[G_{p \circ q} \cap q \circ A\right]\right) .
\end{aligned}
$$

For every $p \in G$ and $\beta>\max (p \circ q)$ we have

$$
\begin{aligned}
\beta & \in \cup\left[G_{p} \cap A\right] \equiv p \cup\{\beta\} \in A \\
& \equiv q \circ(p \cup\{\beta\}) \in q \circ A \equiv(q \circ p) \cup\{\beta\} \in q \circ A \\
& \equiv \beta \in \cup\left[G_{p \circ q} \cap q \circ A\right] .
\end{aligned}
$$

Hence in view of $\kappa$-additivity of $\mu$ we get

$$
\mu\left(\bigcup\left[G_{p} \cap A\right]\right)=\mu\left(\bigcup\left[G_{p \circ q} \cap q \circ A\right]\right)
$$

and finally

$$
m(q \circ A)=\sum_{p \in G} \mu\left(\bigcup\left[G_{p} \cap A\right]\right)=m(A) .
$$


This shows that $m$ is an invariant measure and hence a counterexample to (*) which concludes the proof of our theorem.

An extension of the above argument gives the following

THEOREM 2. Let $(G,+)$ be an abelian group. Then there exists a universal semiregular invariant measure on $(G,+)$ vanishing on singletons iff there exists a real-valued measurable cardinal $\kappa \leqslant|G|$.

Proof. If there exists a measure with the above properties on $(G,+)$ then, as before, there is a subset of $G$ with real-valued measurable cardinality.

In order to show the converse we first consider the case when $(G,+)$ is torsion-free and $|G|=\kappa$ is itself real-valued measurable. Let $\mu$ be a $\kappa$-additive universal finite measure on $\kappa$, vanishing on singletons. We fix a well-ordering $z_{i}: i \in \omega$ of all nonzero integers and for $g \in G$ denote $z_{i} g$ the result of adding $g\left|z_{i}\right|$ times if $z_{i}>0$ and adding $-g\left|z_{i}\right|$ times if $z_{i}<0$.

The group $G$ has a free abelian subgroup $G^{\prime}$ with base of cardinality $\kappa$. Let $x_{\alpha}$ : $\alpha<\kappa$ be an enumeration of this base. Every element $g \in G^{\prime}$ has exactly one representation as $z_{i_{1}} x_{\alpha_{1}}+\cdots+z_{i_{n}} x_{\alpha_{n}}$.

Denote by $\varphi(g)$ the set $\left\{\alpha_{1}, \ldots, \alpha_{n}\right\} \subset \kappa$. Following the idea from the proof of Theorem 1 we put

$$
G_{g}^{i}=\left\{h \in G^{\prime}: \exists \beta>\max (\varphi(g))\left[h=z_{i} x_{\beta}+g\right]\right\},
$$

for $g \in G^{\prime}, i \in \omega$. Now the measure $m: \mathscr{P}\left(G^{\prime}\right) \rightarrow R \cup\{+\infty\}$ is defined as

$$
m(A)=\sum_{i \in \omega} \sum_{g \in G^{\prime}} \mu\left(\bigcup\left(\varphi *\left[G_{g}^{i} \cap A\right]\right)\right) .
$$

The verification that $m$ has all required properties is similar to that in the proof of Theorem 1.

In order to define the measure $m_{1}$ on $G$ we let $\left\{s_{\alpha}+G^{\prime}: \alpha<\lambda\right\}$ be the family of cosets of $G^{\prime}$ in $G\left(\left\{s_{\alpha}: \alpha<\lambda\right\}\right.$ is a fixed selector of this family). On each coset we define $m_{1}$ separately putting $m_{1}\left(s_{\alpha}+A\right)=m(A)$ where $A \subset G^{\prime}$. Since every $A \subset G$ splits into sets $s_{\alpha}+A_{\alpha}: \alpha<\lambda$ where $A_{\alpha} \subset G^{\prime}$ we can define $m_{1}(A)=\Sigma_{\alpha<\lambda} m\left(A_{\alpha}\right)$.

It is easy to see that $m_{1}$ is a $\kappa$-additive semiregular universal measure vanishing on singletons. The only property to verify is invariance. By definition it is enough to check $m_{1}(g+B)=m_{1}(B)$ for $g \in G$ and $B$ included in a coset. Let $g=s_{\alpha}+a$, $a \in G^{\prime}$, and $B=s_{\beta}+A, A \subset G^{\prime}$. We get $m_{1}(g+B)=m_{1}\left(s_{\alpha}+s_{\beta}+a+A\right)$. There exist an element $b \in G^{\prime}$ and an ordinal $\gamma<\lambda$ such that $s_{\alpha}+s_{\beta}=s_{\gamma}+b$. Hence $m_{1}\left(s_{\alpha}+s_{\beta}+a+A\right)=m_{1}\left(s_{\gamma}+b+a+A\right)=m(b+a+A)$. In view of the invariance of $m$ we have $m(b+a+A)=m(A)$ and by definition $m_{1}(B)=m(A)$. Hence finally $m_{1}(g+B)=m_{1}(B)$. This finishes the proof in the case when $(G,+)$ is a torsion-free abelian group of cardinality $\kappa$,

Next we consider the case of arbitrary abelian groups $(G,+)$ of cardinality $\kappa$. Let $H$ be the torsion subgroup of $G$. First assume that $|H|=\kappa$. Then for some $n \in \omega$ the group $H_{n}$ of elements of rank dividing $n$ has cardinality $\kappa$. Let $n_{0}$ be the smallest $n$ with this property. We claim that $n_{0}$ is prime. If not, let $n_{0}=n_{1} n_{2}, 1<n_{1}, n_{2}$. 
There exist $\kappa$ elements $a_{\alpha}: \alpha<\kappa$ of rank exactly $n_{0}$. Consider the family $\left\{n_{1} \cdot a_{\alpha}\right.$ : $\alpha<\kappa\}$. All these elements have rank $n_{2}$ hence there are $<\kappa$ of them. It follows that there are $\kappa$ distinct elements which have equal $n_{1}$-multiples.

Thus there are $\kappa$ elements with rank dividing $n_{1}$ contrary to the minimality of $n_{0}$.

This proves that $n_{0}$ is a prime number and hence the set consisting of elements of rank $n_{0}$ is (together with the neutral element) a group of cardinality $\kappa$. It is also a linear space over the field $Z_{n_{0}}$. Hence we can construct a universal semiregular invariant measure $m_{2}$ on $H_{n_{0}}$ similarly as on a free abelian group. Next we define an extension of this measure to the whole group $(G,+)$ first separately on cosets of $H_{n_{0}}$ in $G$ and then for an arbitrary set as the sum of measures of its intersections with cosets. All details are much the same as in the torsion-free case and hence we leave them to the reader.

If the torsion subgroup $H$ has cardinality $<\kappa$ then $G / H$ is a torsion-free abelian group of cardinality $\kappa$. Hence, by the first part of the proof there exists a universal semiregular invariant measure $m_{3}$ on $G / H$ vanishing on singletons.

Let $U=\left\{u_{\alpha}: \alpha<\kappa\right\}$ be any selector of cosets of $H$ in $G$. The sets $h+U: h \in H$ form a disjoint partition of $G$. On each of them we define the measure $m_{4}$ separately: for $A \subset U$ and $h \in H, m_{4}(h+A)=m_{3}(\{a+H: a \in A\})$.

Finally for an arbitrary $A \subset G$ we put $m_{4}(A)=\Sigma_{h \in H} m_{4}\left(A_{h}\right)$ where $A_{h}=A \cap$ $(h+U)$. Again it is not hard to see that $m_{4}$ is universal, $\kappa$-additive, semiregular and vanishes on singletons. The only property to verify is invariance. In view of $\kappa$-additivity of $m_{4}$ and of $|H|<\kappa$ it is enough to check $m_{4}(a+A)=m_{4}(A)$, for $a \in G$, and $A \subset U$. The set $A$ splits into disjoint sets $\tilde{A}_{k}: h \in H$, such that $a+\tilde{A}_{h} \subset h+U$. Again in view of $\kappa$-additivity it is enough to check $m_{4}\left(a+\tilde{A}_{h}\right)=$ $m_{4}\left(\tilde{A}_{h}\right)$ for each $h \in H$ separately. By definition of $m_{4}$ and invariance of the measure $m_{3}$ we get

$$
\begin{aligned}
m_{4}\left(a+\tilde{A}_{h}\right) & =m_{3}\left(\left\{b+H: b \in a+\tilde{A}_{h}\right\}\right) \\
= & m_{3}\left(\left\{-a+b+H: b \in a+\tilde{A}_{h}\right\}\right)=m_{3}\left(\left\{b+H: b \in \tilde{A}_{h}\right\}\right)=m_{4}\left(\tilde{A}_{h}\right) .
\end{aligned}
$$

This proves the invariance of $m_{4}$ and finishes the proof in the case when $(G,+)$ is an abelian group with real-valued measurable cardinality.

In the general case let $\kappa \leqslant|G|$ be a real-valued measurable cardinal and let $H$ be a subgroup of $G$ of cardinality $\kappa$. We construct a measure on $H$ with the required properties and then extend it to $G$ as described in the previous part of the proof.

REMARK. The above constructed measure has the following additional property: any set $A$ has the same measure as $\{-a: a \in A\}$.

PROBLEM. Is Theorem 2 true for arbitrary groups?

\section{REFERENCES}

1. F. Drake, Set theory. An introduction to large cardinals, North-Holland, Amsterdam, 1974.

2. P. Erdös and R. D. Mauldin, The nonexistence of certain invariant measures, Proc. Amer. Math. Soc. 59 (1976), 321-322.

3. V. Kannan and S. Radhakrishnesvara Raju, The nonexistence of invariant universal measures on semigroups, Proc. Amer. Math. Soc. 78 (1980), 482-484. 